Mens

revue d'histoire intellectuelle de l'Amérique française

\title{
L'histoire des droites européennes : bibliographie sélective
}

\section{Christian Roy}

Volume 6, numéro 2, printemps 2006

URI : https://id.erudit.org/iderudit/1024314ar

DOI : https://doi.org/10.7202/1024314ar

Aller au sommaire du numéro

Éditeur(s)

Centre de recherche en civilisation canadienne-française

ISSN

1492-8647 (imprimé)

1927-9299 (numérique)

Découvrir la revue

Citer ce document

Roy, C. (2006). L'histoire des droites européennes : bibliographie sélective.

Mens, 6(2), 313-336. https://doi.org/10.7202/1024314ar d'utilisation que vous pouvez consulter en ligne.

https://apropos.erudit.org/fr/usagers/politique-dutilisation/ 


\title{
Bibliographie
}

\section{L'HISTOIRE DES DROITES EUROPÉENNES : BIBLIOGRAPHIE SÉLECTIVE}

\author{
Cbristian Roy \\ Facultés de théologie et de philosophie \\ Université Laval
}

L'histoire intellectuelle d'un ensemble disparate de familles politiques nationales et transnationales à l'échelle du continent européen se laisse difficilement distinguer de l'histoire tout court des multiples courants qui le composent, ni de leur analyse sociologique ou proprement politologique. C'est peut-être plus vrai encore de «la » droite que de "la " gauche. Car si la seconde, basée sur une volonté de changement, est toujours portée par des idéologies consciemment articulées, le propre de la première est de contrer celles-ci de façon plus réactive, en faisant appel tantôt à un héritage antérieur de représentations traditionnelles (souvent demeurées implicites jusqu'à leur contestation moderne), tantôt au dynamisme d'un mouvement visant à recréer sur le terrain de la modernité l'équivalent de l'unité perdue d'un état de civilisation du passé, tantôt encore au scepticisme proclamé envers toute idéologie au nom de la sagesse pragmatique de la croissance évolutive des institutions au gré des changements graduels de la société, sans autre téléologie que la viabilité de sa reconduction. 
Dans tous les cas, le rôle de la théorie en tant que telle tend à être beaucoup moins important pour les droites que pour les gauches, de telle sorte que la proportion du traitement des idées par rapport au traitement du phénomène social tend à s'inverser entre leurs historiographies respectives (Suvanto, 1997, p. 1). Ainsi les idées sont-elles souvent plus diffuses et moins aptes à être isolées de leur contexte sociologique dans le cas de bien des droites. Raison de plus pour en proposer ici une bibliographie générale, non limitée à leur composante intellectuelle explicite, mais lui faisant bien sûr la plus large place, sans exclure les anthologies et études où des auteurs de droite passent en revue diverses traditions de droite.

En guise d'introduction à ce champ historiographique foisonnant aux frontières internes et externes fluctuantes, je tenterai plus loin de distinguer les principales de ces traditions. Mais peut-être convient-il d'abord de préciser la notion de conservatisme qui en traverse la plupart (sauf certaines acceptions libertariennes plutôt liées aux États-Unis malgré des sources autrichiennes), et qui s'attache plus particulièrement à ses plus anciennes formes : la droite traditionaliste et la droite modérée. L'attachement conservateur à un héritage du passé trouve à droite trois principaux motifs de justification : la solidarité organique d'une société d'états, la défense de la propriété dans une société de classes, et le scepticisme envers toute intervention volontariste de la raison humaine dans la vie sociale. Dans la tradition du conservatisme britannique où leur confrontation est la plus explicite, les deux premiers motifs, correspondant respectivement au courant tory et au courant whig, tendent chacun à s'appuyer sur l'empirisme anti-idéologique du troisième, invoquant la caution de penseurs allant de David Hume à Michael Oakeshott. Selon le courant favorisé par tel ou tel auteur, l'historiographie bri- 
tannique du conservatisme est largement partagée entre la tendance à exclure du canon les penseurs et acteurs qui ne s'inscrivent pas dans la tendance tenue pour authentique, ou au contraire, à y inclure anachroniquement et sélectivement des figures étrangères à celle-ci, voire à certains égards à la tradition du conservatisme historique - comme par exemple le Whig Edmund Burke, désigné rétrospectivement comme fondateur du conservatisme moderne par tous ses tenants. Il est vrai que leur adoption de Burke se base sur son rejet réfléchi de la Révolution française, repoussoir commun aux trois courants du conservatisme britannique. Qu'est-ce qui unit en revanche pragmatistes doutant du pouvoir des idées, patriciens romantiques convaincus que noblesse oblige et néo-libéraux puritains jurant par la discipline du marché ? Et qu'estce qui distancie ces derniers de la tradition libérale proprement dite?

It is the persistent image of society as a command structure in which the responsibilities of leadership can be exercised within the framework of a strong state manifested in divine-right royalism, the Tory conception of mixed sovereignty, anti-Jacobinism, the doctrine of the survival of the fittest, One Nation Toryism and so forth - that distinguishes English conservatism from rival ideologies (Eccleshall, 1990, pp. 18-19).

C'est aussi ce qui unit cette idéologie britannique à la droite continentale telle qu'elle prend forme en France durant la Révolution à l'occasion des débats de l'Assemblée nationale de la fin de l'été 1789 , lorsque les députés favorables au veto royal se concentrent à la droite de l'hémicycle parlementaire, qui fournit dès lors la matrice de l'éventail politique de l'Europe contemporaine. Tandis que la cohabitation plus ou moins tendue d'éléments whigs et torys en un parti conservateur prend forme au Royaume-Uni à partir du second tiers du $\mathrm{XIX}^{\mathrm{e}}$ siècle, de la France de la Restauration 
(où Chateaubriand lance le journal Le Conservateur en 1818), l'orientation et la terminologie "droite / gauche » se propagent dans certaines principautés allemandes dès avant les révolutions de 1848, puis au Danemark en 1870, à la Norvège en 1882, à la Suède en 1910 et au Luxembourg en 1914, (Atkin et Tallett, 2003, pp. 1-2). C'est à cette époque que remontent en France, au confluent de l'histoire et de la science politique, les premières études pséphologiques d'André Siegfried y analysant rétrospectivement les familles de droite et de gauche. Le relais sera pris après la Seconde Guerre mondiale par deux historiens démocrates-chrétiens, le protestant François Goguel et le catholique René Rémond (Sirinelli, 1992, XVXXXVI). Ce dernier surtout consacre à la droite une monographie qui demeure le point de départ de son étude générale pour l'Europe continentale. La droite en France de 1815 à nos jours (1954) retrace les incarnations successives de ses trois traditions d'origine : le légitimisme (réactionnaire intransigeant), l'orléanisme (conservateur modéré) et le bonapartisme (national-populiste). La troisième édition, Les droites en France (1982), fait écho aux critiques adressées à cette élégante thèse qui tendait à minimiser la nouveauté historique de certains courants et notamment à nier qu'il eût existé un fascisme français de quelque importance. Or dès les années 1960, l'Action française royaliste de Charles Maurras avait déjà droit à son propre volume dans la trilogie d'Ernst Nolte sur Le Fascisme dans son époque, suivie des études d'Eugen Weber sur ces mêmes thèmes ainsi que par le grand panorama historique de la droite européenne dans son ensemble et dans toute la diversité de ses traditions nationales qu'il publia avec Hans Rogger ; The European Right: A Historical Profile (1966) demeure sans doute la meilleure introduction générale à l'étude comparative des droites. 
Si la droite avait à cette époque un cachet d'exotisme sulfureux (bien rendu par la couverture noire de l'excellente collection de sources "Roots of the Right»), elle allait commencer à susciter un intérêt sérieux, se traduisant par une multiplication des publications, à partir de la fin des années 1970. C'est que s'estompe alors le consensus bicéphale marxokeynésien des décennies d'après-guerre et que se manifeste inopinément, à la faveur de la crise des modèles de la gauche et du centre, ce qu'il est convenu d'appeler une "Nouvelle Droite ». Il serait en fait plus exact d'user du pluriel puisque cette expression a pu servir à désigner au moins trois courants bien distincts et souvent hostiles les uns aux autres : la New Right néo-libérale d'origine anglo-saxonne, et à partir de la France, de nouveaux partis populistes xénophobes tels le Front National en même temps qu'une Nouvelle Droite intellectuelle antiatlantiste et euro-tiers-mondiste, communautariste et néopaïenne, répondant à la Nouvelle Gauche par les moyens culturels d'un "gramscisme de droite ". L'initiateur de ce dernier mouvement qui a trouvé des émules et des épigones plus ou moins fidèles de l'Espagne à la Russie, Alain de Benoist, a aussi contribué par ses nombreux travaux d'érudition (notamment bibliographiques) à rendre accessibles de vastes pans du patrimoine intellectuel des droites de France et d'Allemagne.

C'est encore surtout pour le pays où elle fut d'abord nommée et conceptualisée que la droite trouve des études d'ensemble ambitieuses telles que la monumentale trilogie dirigée par Jean-François Sirinelli sur l'Histoire des droites en France (1992), particulièrement attentive aux relais institutionnels et culturels des différents discours de droite. Cette somme témoigne de l'essor général de l'étude des droites à l'échelle occidentale à la fin du siècle dernier. À titre d'exemples, à côté des panoramas européens de l'extrême droite, de la dé- 
mocratie chrétienne et du libéralisme parus en Belgique dans la collection "Questions au $\mathrm{XX}^{\mathrm{e}}$ siècle » des Éditions Complexe, on trouve aussi de grandes synthèses comparatives du conservatisme comme celles du Grec Panayotis Kondylis et du Finlandais Pekka Suvanto, ou encore la collection "Twayne's Themes in Right-Wing Politics and Ideology ». C'est de l'un de ses volumes que je tire la grille de classification qui me paraît la plus utile pour tenter de distinguer les principaux types de droites entre lesquelles peuvent se répartir les traditions intellectuelles de ce bord, même si certaines de ces catégories se recoupent forcément au gré des positionnements et parcours de leurs représentants, et sont souvent amalgamées au gré des aires d'étude et des angles d'approche de leurs interprètes. C'est pour cette raison que j'ai dû me contenter de grouper dans cette bibliographie d'une part celles nées au $\mathrm{XIX}^{\mathrm{e}}$ siècle : la droite réactionnaire et la droite modérée, et d'autre part celles surgies au $\mathrm{XX}^{\mathrm{e}}$ siècle : droite radicale, extrême droite et nouvelles droites.

Roger Eatwell a en effet pu distinguer cinq «styles de pensée » de droite surgis de la critique du libéralisme et du socialisme : une droite réactionnaire, une droite modérée, une droite radicale, une droite extrême et une nouvelle droite entendue aux sens contradictoires des pensées néo-libérales du marché généralisé d'une part et des pensées communautaristes de l'enracinement culturel d'autre part. Ces deux nouvelles droites ont pourtant en commun de vouloir refaçonner la nature humaine - la première en fonction de la pensée rationnelle utilitariste de l'bomo economicus et la seconde en fonction de la pensée mythique antiutilitariste de l'bomo politicus. Or les droites les plus anciennes se méfiaient précisément de toute velléité révolutionnaire de refaire l'homme, la droite réactionnaire parce qu'elle croyait à l'ordre immuable d'un ancien régime idéalisé aux fondements théologiques ab- 
solus et objectifs, et la droite modérée parce qu'elle croyait à l'évolution graduelle des sociétés dans le contexte historique de leur expérience propre. Si la première est caractérisée par l'intransigeance et l'intolérance et ne se survit plus guère que dans quelques ghettos intégristes, la seconde recherche un équilibre entre la continuité et le changement et admet donc le pluralisme et l'alternance, ce qui lui a assuré une large postérité dans le système des partis de la démocratie libérale. La polarisation droite-gauche aidant, il est même possible de grouper conservatisme et libéralisme sous la même étiquette de centre-droit, puisque ces idéologies partagent beaucoup de valeurs, de références et de pratiques. On a vu qu'au RoyaumeUni, elles ont historiquement coexisté au sein du parti conservateur.

Elsewhere in Europe, the center-right is fragmented into two or more parties representing one or more traditions or ideologies: classical conservatism, mercantilism, anticommunism, pragmatism, nationalism, Christian democracy, and historical liberalism (Wilson, 1998, p. ix).

Eatwell distingue encore la droite radicale de la droite extrême, que beaucoup tendent à confondre. Surgie en réponse au socialisme, la droite radicale prétend rivaliser avec lui sur le terrain de l'activisme et s'oppose à la réaction en ce qu'elle cherche à apporter des solutions originales aux problèmes de la modernité, sans craindre de créer de toutes pièces le nouvel état social plus "organique » jugé souhaitable. Son expression la plus intéressante est la nébuleuse de courants intellectuels germaniques contestataires de la République de Weimar groupée par le Suisse Armin Mohler sous la rubrique commune de "Révolution conservatrice" dans sa thèse d'abord publiée en 1950, transformée en manuel universitaire en 1972, rééditée en deux volumes en 1989, et en- 
fin traduite en 1993 à l'initiative d'Alain de Benoist. Celui-ci a aussi contribué une "Bibliographie française de la Révolution conservatrice allemande » à ce projet de première importance pour la Nouvelle Droite intellectuelle française qui perpétue cette tradition : le GRECE (Groupe de recherches et d'études sur la civilisation européenne). La " Nouvelle Droite » politique du Front national de Jean-Marie Le Pen offre en revanche un bon exemple de droite extrême, moins soucieuse de théorie critique que de théories de complots, mieux à même de mobiliser les masses contre des boucs émissaires désignés (étrangers, communisme, etc.).

These arguments are usually presented in crude form; indeed, a defining characteristic of the extreme right is the paucity of its intellectual tradition. The reactionary, moderate and radical rights have produced significant political theorists. The extreme right has tended more to produce propagandists, interested in telling people what to think rather than how to think, and lacking in originality. The radical right was genuinely interested in how to achieve the good citizen; the extreme right has been more manipulative, paranoid (Eatwell \& O'Sullivan, 1989, p. 71).

Cette distinction peut aider à situer les différents fascismes et courants du national-socialisme par rapport à la droite radicale et à l'extrême droite. Mais lesquelles des droites radicales et extrêmes peuvent être considérées comme fascistes ? Le fascisme est-il un phénomène de droite, ou brouille-t-il la distinction droite-gauche par son caractère révolutionnaire et ses sources socialistes ? Si le fascisme et les droites radicales et extrêmes se disent parfois " ni de droite ni de gauche ", cela implique-t-il que quiconque dit «ni droite ni gauche» est de droite ou fasciste? Au cœur des débats agitant l'historiographie très riche et complexe du fascisme, ces questions soulevées depuis les années 1980 par les travaux de Zeev Sternhell 
sur ses sources intellectuelles françaises mettent en cause les frontières de l'historiographie de la droite, puisque les réponses qu'il apporte tendent à lui donner une extension indéfiniment élastique en conceptualisant le fascisme comme un « extrême centre » qui se ramène malgré tout à l'extrême droite, et en y incluant ce qu'il est convenu d'appeler, depuis la thèse classique de Jean-Louis Loubet del Bayle (d'abord parue en 1969), le "non-conformisme " de mouvements politiques contestataires ayant pris entre les deux guerres en France une certaine distance par rapport aux familles de droite et de gauche dont ils étaient souvent issus. Est-il bien légitime de situer comme ce dernier la Jeune Droite post-maurrassienne (que l'on connaît mieux depuis les récents travaux de Nicolas Kessler et de Véronique Auzépy-Chavagnac) non seulement parmi l'éventail des droites, mais à l'intérieur d'un continuum autonome de positions indépendantes, avec les personnalismes de la revue Esprit penchant à gauche et du groupe Ordre Nouveau qui n'a jamais désavoué sa ligne non-conformiste " ni droite ni gauche», et dont le cas est aggravé par sa malencontreuse homonymie avec le slogan préféré de générations d'idéologues de droite ? Si c'est certes une question qui se pose à l'historiographie de la droite intellectuelle de nos jours, je dois avouer être de ceux qui lui apportent sans ambages une réponse positive, réclamant la consolidation d'un champ historiographique distinct pour les non-conformismes ou « nouvelles relèves ", comme les appelle Olivier Dard. Avec ce dernier et le sociologue Martin Meunier, je cherche d'ailleurs à constituer un réseau international de chercheurs sur ce sujet. Étant plus particulièrement chargé de la compilation de sa bibliographie pour les Amériques, je me permets en terminant de lancer un appel à tout lecteur qui aurait quelque contribution à apporter à ce projet visant à explorer les réponses politiques aux défis de la modernité qui ont remis en cause l'axe gauche-droite. 


\section{Les droites en général}

***. «La droite : Idéologies et littérature », dossier du Magazine littéraire, $\mathrm{n}^{\circ}$ 305, (décembre 1992).

ALEXANDRE-COLIER, Agnès et Xavier JARDIN. Anatomie des droites européennes. Paris, Armand Colin, 2004.

ATKIN, Nicholas et Frank TALLETT. The Right in France: from Revolution to Le Pen. Londres et New York, I. B. Tauris, 2003.

BENOIST, Alain de. Bibliographie générale des droites françaises. Coulommiers, Dualpha, 2004.

Tome 1 : Arthur de Gobineau, Gustave Le Bon, Édouard Drumont, Maurice Barrès, Pierre Drieu La Rochelle, Henry de Montherlant, Thierry Maulnier, Julien Freund. Tome 2: Georges Sorel, Charles Maurras, Georges Valois, Abel Bonnard, Henri Béraud, Louis Rougier, Lucien Rebatet, Robert Brasillach. Tome 3: Louis de Bonald, Joseph de Maistre, Alexis de Tocqueville, Georges Vacher de Lapouge, Charles Péguy, René Benjamin, Georges Bernanos, Jean Cau. Tome 4 : Ernest Renan, Jules Soury, Léon Daudet, Henri Massis, Jacques Bainville, Jacques Benoist-Méchin, Marc Augier, Louis Pauwels.

EATWELL, Roger et Noël O'SULLIVAN, dirs. The Nature of the Right: American and European Politics and Political Thought since 1789. Boston, Twayne's Publishers, 1989. (Coll. "Twayne's Themes in Right-Wing Politics and Ideology Series $")$.

GIRVIN, Brian. The Right in the Twentieth Century. Conservatism and Democracy. Boston, Twayne's Publishers, 1994. (Coll. "Twayne's Themes in Right-Wing Politics and Ideology Series "). 
LAQUEUR, Walter. Histoire des droites en Russie. Des centuries noires aux nouveaux extrémistes. Paris, Michalon, 1996.

MATTIOLI, Aram, dir. Intellektuelle von rechts. Ideologie und Politik in der Schweiz 1918-1939. Zurich, Orell Fussli, 1995.

McCLELLAND, J. S., dir. The French Right (from de Maistre to Maurras). New York, Harper \& Row, 1970. (Coll. « Roots of the Right $)$.

PETITFILS, Jean-Christian. La droite en France de 1789 à nos jours. Paris, Presses universitaires de France, 1989. (Coll. "Que sais-je?»).

RÉMOND, René. La droite en France de 1815 à nos jours. Continuité et diversité d'une tradition politique. Paris, Aubier, 1954.

RÉMOND, René. Les droites en France. Paris, Aubier, 1982. RICHARD, François. L'anarchisme de droite dans la littérature contemporaine. Paris, Presses universitaires de France, 1988. (Coll. «Littératures modernes »).

RICHARD, Gilles et Jacqueline SAINCLIVIER, dirs. La recomposition des droites en France à la Libération, 1944-1948. Rennes, Presses universitaires de Rennes, 2004.

ROGGER, Hans et Eugen WEBER, dirs. The European Right. A Historical Profile. Berkeley et Los Angeles, University of California Press, 1966.

SHAPIRO, David M. The Right in France, 1890-1919. Three Studies. Londres, Chatto \& Windus, 1962. 
SIRINELLI, Jean-François, dir. Histoire des droites en France.

Paris, Gallimard, 1992.

Tome 1 : Politique.

Tome 2: Cultures.

Tome 3: Sensibilités.

WEBBER, G. C. The Ideology of the British Right 1918-1939.

New York, St. Martin's Press, 1986.

WINOCK, Michel, dir. La Droite depuis 1789. Les hommes, les idées, les réseaux. Paris, Seuil, 1995.

\section{Droites réactionnaires et droites modérées}

***. De Gaulle et Malraux. Colloque organisé par l'Institut Charles-de-Gaulle les 13, 14 et 15 novembre 1986. Paris, Plon, 1987. (Coll. «Espoir»).

ALBA, Víctor. Los conservadores en España : ensayo de interpretación bistórica. Barcelone, Planeta, 1981.

ALTERMATT, Urs. "Conservatism in Switzerland: A Study in Antimodernism ». Journal of Contemporary History, vol. 14, (1979) ("A Century of Conservatism, Part $2 »)$, pp. 581-610.

ANDERSON, Malcolm. Conservative Politics in France. Londres, Allen \& Unwin, 1974.

AUGHEY, Arthur, Greta JONES et W. T. M. RICHES. The Conservative Political Tradition in Britain and the United States. Londres, Pinter Publishers, 1992.

BARNES, John et al., dirs. A History of the Conservative Party. Londres et New York, Longman, 1978-1979. Tome 1 : Robert STEWART. The Foundation of the Conservative Party 1830-1867. 
Tome 2 : Paul ADDISON. The Rise of Tory Democracy $1867-$ 1902.

Tome 3 : John RAMSDEN. The Age of Balfour and Baldwin 1902-1940.

Tome 4 : John BARNES. From Affluence to Disillusion 19401974.

BLAKE, Robert. The Conservative Party from Peel to Churchill. Londres, Eyre \& Spottiswoode, 1970.

BLINKHORN, Martin, dir. Fascists and Conservatives. The Radical Right and the Establishment in Twentieth-Century Europe. Londres et Boston, Unwin Hyman, 1990.

BLUCHE, Frédéric. Le Bonapartisme : aux origines de la droite autoritaire (1800-1850). Paris, Nouvelles éditions latines, 1980.

BUTLER, Lord, dir. The Conservatives. A History from their Origins to 1965. Londres, George Allen \& Unwin, 1977. CHARLOT, Jean. Le phénomène gaulliste. Paris, Fayard, 1970. CHARMELY, John. A History of Conservative Politics, 19001996. New York, St. Martin's Press, 1996.

COLEMAN, Bruce. Conservatism and the Conservative Party in Nineteenth-Century Britain. Londres, Edward Arnold, 1988.

CONWAY, Martin, Catbolic Politics in Europe 1918-1945. Londres et New York, Routledge, 1997.

DU PUY DE CLINCHAMPS, Philippe. Le royalisme. $2^{\mathrm{e}}$ éd. mise à jour par Jean-Paul Alexis. Paris, Presses universitaires de France, 1981. (Coll. "Que sais-je ?»).

DURAND, Jean-Dominique. L'Europe de la démocratie cbrétienne. Bruxelles, Complexe, 1995. (Coll. "Questions au $\mathrm{XX}^{\mathrm{e}}$ siècle $川$ ). 
ECCLESHALL, Robert. English Conservatism since the Restoration. Londres, Unwin Hyman, 1990.

EINAUDI, Mario et François GOGUEL. Christian Democracy in Italy and France. Notre Dame, University of Notre Dame Press, 1952.

EPSTEIN, Klaus. The Genesis of German Conservatism. Princeton, Princeton University Press, 1976.

EVANS, Ellen L. The Cross and the Ballot. Catholic Political Parties in Germany, Switzerland, Austria, Belgium and the Netherlands, 1785-1985. Boston, Humanities Press, 1999.

FLAMANT, Maurice. Histoire du libéralisme. Paris, Presses universitaires de France, 1992. (Coll. "Que sais-je ?»).

GENGEMBRE, Gérard. La Contre-Révolution ou l'bistoire désespérante. Paris, Imago, 1989.

GIRARDET, Raoul. Le Nationalisme français, 1871-1914. Paris, Colin, 1966.

GRAY, John. Liberalism. Minneapolis, University of Minnesota Press, 1995.

GRIFFITHS, Richard. The Reactionary Revolution. The Catbolic Revival in French Literature, 1870-1914. Londres, Constable, 1966.

HANLEY, David, dir. Christian Democracy in Europe. $A$ Comparative Perspective. Londres et New York, Pinter, 1996.

HEARNSHAW, F. J. C. Conservatism in England: An Analytical, Historical, and Political Survey. Londres, Macmillan \& Co., 1933 (réimpression : New York, H. Fertig, 1968).

HIRSCHMAN, Albert O. The Rhetoric of Reaction: Perversity, Futility, Jeopardy. Cambridge (Mass.), Belknap Press, 1991. 
HOLMES, Stephen. Benjamin Constant and the Making of Modern Liberalism. New Haven, Yale University Press, 1984. IRVINE, William Drummond. French Conservatism in Crisis: The Republican Federation of France in the 1930s. Baton Rouge, Louisiana State University Press, 1979.

IRVING, R. E. M. Christian Democracy in France. Londres, Allen \& Unwin, 1973.

JARDIN, André. Histoire du libéralisme politique, de la crise de l'absolutisme à la constitution de 1875. Paris, Hachette, 1985.

KAISER, Wolfram et Michael GEHLER, dir. Christian Democracy in Europe since 1945. Portland, Frank Cass, 2004.

KIRCHNER, E. J., dir. Liberal Parties in Western Europe. Cambridge, Cambridge University Press, 1988.

KONDYLIS, Panayotis. Konservativismus : geschichtlicher Gehalt und Untergang. Stuttgart, Klett-Cotta, 1986.

KUKATHAS, Chandran. Hayek and Modern Liberalism. Londres, Clarendon Press, 1990.

LAMBERTS, Emiel, dir. Cbristian Democracy in the European Union, 1945-1995: Proceedings of the Leuven Colloquium, 15-18 November 1995. Louvain, Leuven University Press, 1997.

LUDWIKOWSKI, Rett R. Continuity and Change in Poland: Conservatism in Polish Political Thought. Washington, Catholic University of America Press, 1991.

LYTTELTON, Adrian, dir. Liberal and Fascist Italy: 1900 1945. Oxford et New York, Oxford University Press, 2002.

MANNHEIM, Karl. Conservatism: A Contribution to the Sociology of Knowledge. Londres et New York, Routledge \& Kegan Paul, 1986. 
O'GORMAN, Frank. British Conservatism. Conservative Thought from Burke to Thatcher. Londres et New York, Longman, 1986.

O’SULLIVAN, Noël. Conservatism. Londres, J. M. Dent \& Sons, 1976.

PAPINI, Roberto, The Christian Democrat International. Lanham, Rowman \& Littlefield Publishers, 1997.

QUINTON, Anthony. The Politics of Imperfection. The Religious and Secular Traditions of Conservative Thought in England from Hooker to Oakeshott. Londres et Boston, Faber \& Faber, 1978.

RENOUVIN, Bertrand. Le royalisme: Histoire et actualité. Paris, Economica, 1997.

RIALS, Stéphane. Le légitimisme. Paris, Presses universitaires de France, 1983. (Coll. "Que sais-je?»).

RIALS, Stéphane. Les idées politiques du Président Georges Pompidou. Paris, Presses universitaires de France, 1977. ROUSSELLIER, Nicolas. L'Europe des libéraux. Bruxelles, Complexe, 1991. (Coll. "Questions au XX' siècle»). SCHUETTINGER, Robert L., dir. The Conservative Tradition in European Thought. New York, G. P. Putnam's, 1975.

SCHUMANN, Hans-Gerd. Konservativismus. Königstein, Athenäum, 1984.

SCRUTON, Roger, dir. Conservative Texts. An Anthology. Londres, Macmillan, 1991.

SCRUTON, Roger. Conservative Thinkers. Londres, Claridge Press, 1988. 
SECO SERRANO, Carlos. Historia del conservadurismo español: una linea política integradora en el siglo XIX. Madrid, Temas de hoy, 2000.

SIDORSKY, David, dir. The Liberal Tradition in European Thought. New York, Putnam, 1970.

SUVANTO, Pekka. Conservatism from the French Revolution to the 1990s. New York, St. Martin's Press, 1997.

TOUCHARD, Jean. Le Gaullisme 1940-1969. Paris, Seuil, 1978.

VEEN, Hans-Joachim, dir. Christlich-demokratische und konservative Parteien in Westeuropa. Paderborn, F. Schöningh, Tome 1: CDU, 1983.

Tome 2: Grossbritannien, 1983.

Tome 3 : Italien, Griechenland. Rudolf Lill, 1991.

Tome 4 : Scbweden, Norwegen, Finnland, Dänemark. Bernd Henningsen, 1994.

Tome 5: Scbweiz, Niederlande, Belgien, Luxemburg, Europäische Demokratische Union (EDU), Europäische Volkspartei (EVP). Urs Altermatt, 2000.

WEBSTER, Richard A. Christian Democracy in Italy, 18601960. Londres, Hollis \& Carter, 1961.

WEBSTER, Richard A. The Cross and the Fasces. Christian Democrayy and Fascism in Italy. Stanford, Stanford University Press, 1960.

WEISS, John. Conservatism in Europe 1770-1945:

Traditionalism, Reaction and Counter-Revolution. New York, Harcourt Brace Jovanovich, 1977.

WHITE, R. J. The Conservative Tradition. Londres, A. \& C. Black, 1964. 
WILSON, Frank L., dir. The European Center-Right at the End of the Twentieth Century. New York, St. Martin's Press, 1998.

\section{Droites radicales, extrêmes-droites et " nouvelles droites »}

ALGAZY, Joseph. L'extrême droite en France de 1965 à 1984. Paris, L'Harmattan, 1989.

ALGAZY, Joseph. La tentation néo-fasciste en France 19441965. Paris, Fayard, 1984.

ARNOLD, Edward J. The Development of the Radical Right in France: from Boulanger to Le Pen. New York, St. Martin's Press, 2000.

AUZÉPY-CHAVAGNAC, Véronique. Jean de Fabrìgues et la Jeune Droite Catbolique. Aux sources de la Révolution nationale. Villeneuve d'Ascq, Presses universitaires du Septentrion, 2002.

BALACE, Francis, dir. De l'avant à l'après-guerre : l'extrême droite en Belgique francophone. Bruxelles, De Boeck Université, 1994.

BENOIST, Alain de. $V u$ de droite. Anthologie critique des idées contemporaines. Paris, Copernic, 1977.

BLINKHORN, Martin, dir. Fascists and Conservatives. The Radical Right and the Establishment in Twentieth-Century Europe. Londres et Boston, Unwin Hyman, 1990.

BREUER, Stefan. Anatomie de la révolution conservatrice. Paris, Éditions de la Maison des Sciences de l'Homme, 1996.

CARSTEN, Francis L. Fascist Movements in Austria: From Scbönerer to Hitler. Londres et Beverly Hills, SAGE Publications, 1997. 
CHELES, Luciano, Ronnie FERGUSON et Michalina VAUGHN. The Far Right in Western and Eastern Europe. Harlow, Longman, 1995. 2 vol.

CHEBEL D'APPOLLONIA, Ariane. L'extrême droite en France: de Maurras à Le Pen. Bruxelles, Complexe, 1988. (Coll. "Questions au $\mathrm{XX}^{\mathrm{e}}$ siècle »).

COSTA PINTO, Antonio. Salazar's Dictatorship and European Fascism. New York, Columbia University Press, 1995.

CRONIN, Mike. The Blueshirts and Irish Politics. Dublin et Portland, Four Courts Press, 1997.

DARD, Olivier. Le Rendez-vous manqué des relèves des années 30. Paris, Presses universitaires de France, 2002.

DAVIES, Peter et Derek LYNCH. The Routledge Companion to Fascism and the Far Right. Londres et New York, Routledge, 2002.

DE FELICE, Renzo. Les interprétations du fascisme. Paris, Les Syrtes, 2000.

DELWIT, Pascal, Jean-Michel DE WAELE et Andrea REA. L'extrême droite en France et en Belgique. Bruxelles, Complexe, 1998.

DURANTON-CRABOL, Anne-Marie. L'Europe de l'extrême droite de 1945 à nos jours. Bruxelles, Complexe, 1991. (Coll. "Questions au XX $\mathrm{XX}^{\mathrm{e}}$ siècle »).

DURANTON-CRABOL, Anne-Marie. Visages de la Nowvelle Droite : le GRECE et son histoire. Paris, Presses de la Fondation nationale des Sciences politiques, 1988.

EATWELL, Roger. Fascism: A History. Londres, Vintage, 1996. 
ELEY, Geoffrey. Reshaping the German Right: Radical Nationalism and Political Change after Bismarck. Ann Arbor, University of Michigan Press, 1990.

GENTILE, Emilio. Qu'est-ce que le fascisme? Histoire et interprétation. Paris, Gallimard, 2004.

GRIFFIN, Roger, dir. International Fascism: Theories, Causes and the New Consensus. Londres et New York, Oxford University Press, 1998.

HAMILTON, Alastair. Lillusion fasciste. Les intellectuels et le fascisme, 1919-1945. Paris, Gallimard, 1973.

HERF, Jeffrey. Reactionary Modernism. Technology, Culture, and Politics in Weimar and the Third Reich. Cambridge, Cambridge University Press, 1984.

JENKINS, Brian, dir. France In The Era of Fascism. Essays on the French Authoritarian Right. New York et Oxford, Berghahn Books, 2005.

JOST, Hans Ulrich. Les Avant-gardes réactionnaires. La naissance de la nowvelle droite en Suisse (1890-1914). Lausanne, Éditions d'en bas, 1992.

KESSLER, Nicolas. Histoire politique de la Jeune Droite (19291942). Une révolution conservatrice à la franşaise. Paris, L'Harmattan, 2001.

KITCHEN, Martin. The Coming of Austrian Fascism. Montréal, McGill-Queen's University Press, 1980.

KLEMPERER, Klemens von. Germany's New Conservatism: Its History and Dilemma in the Twentieth Century. Princeton, Princeton University Press, 1968.

LAQUEUR, Walter, dir. Fascism. A Reader's Guide. Analyses, Interpretation, Bibliography. Londres, Wildwood House, 1976. 
LARSEN, Stein Ugelvik et al. Who Were the Fascists. Social Roots of European Fascism. Bergen, Universitetsforlaget, 1980.

LOUBET DEL BAYLE, Jean-Louis. Les Non-conformistes des années 30. Une tentative de renowvellement de la pensée politique française. Édition revue et actualisée par l'auteur. Paris, Seuil, 2001.

LYTTELTON, Adrian, dir. Italian Fascisms from Pareto to Gentile. New York, Harper \& Row, 1975. (Coll. «Roots of the Right $)$.

LYTTELTON, Adrian, dir. Liberal and Fascist Italy: 19001945. Oxford et New York, Oxford University Press, 2002. MANNING, Maurice. The Blueshirts. Dublin, Macmillan, 1970.

MILZA, Pierre. Fascisme français. Passé et présent. Paris, Flammarion, 1987.

MOHLER, Armin. La révolution conservatrice en Allemagne (1918-1932). Puiseaux, Pardès, 1993. (Coll. «Révolution conservatrice $")$.

MOREAU, Patrick. Les béritiers du III ${ }^{e}$ Reich : l'extrême droite allemande de 1945 à nos jours. Paris, Seuil, 1994.

MUTTI, Claudio. Les plumes de l'archange. Quatre intellectuels roumains face à la Garde de Fer: Nae Ionescu, Mircea Eliade, Emil Cioran, Constantin Noica. Chalon-sur-Saône, Hérode, 1993.

NAGY-TALAVERA, Nicholas M. The Green Shirts and the Others: $A$ History of Fascism in Hungary and Rumania. Lasi et Portland, Center for Romanian Studies, 2001. 
NGUYEN, Victor. Aux origines de l'Action fransaise. Intelligence et politique à l'aube du XX'e siècle. Paris, Fayard, 1991. NOLTE, Ernst. Le fascisme dans son époque. Paris, Julliard, 1970 ,

Tome 1 : L'Action francaise.

Tome 2: Le Fascisme italien.

Tome 3: Le National-socialisme.

NOLTE, Ernst. Les fondements bistoriques du National-socialisme. Monte Carlo, Le Rocher, 2002.

OSTENC, Michel. Intellectuels italiens et fascisme 1915-1929. Paris, Payot, 1983.

PAXTON, Robert. Le Fascisme en action. Paris, Seuil, 2004.

PAYNE, Stanley G. Fascism in Spain, 1923-1977. Madison, University of Wisconsin Press, 1999.

PAYNE, Stanley G. A History of Fascism, 1914-1945. Madison, University of Wisconsin Press, 1999.

PETITFILS, Jean-Christian. L'extrême droite en France. Paris, Presses universitaires de France, 1988. (Coll. "Que saisje ? »).

POMEYROLS, Catherine et Claude HAUSER, dir. L'Action française et l'étranger: usages, réseaux et représentations de la droite nationaliste française. Paris, L'Harmattan, 2001.

REES, Philip. Fascism and Pre-Fascism in Europe 1890-1945: A Bibliography of the Extreme Right. Brighton (Sussex) et Totowa, Harvester Press et Barnes \& Noble, 1985. 2 vol.

RENARD, Paul. L'Action française et la vie littéraire (19311944). Villeneuve d'Ascq, Presses universitaires du Septentrion, 2003. 
RINTALA, Marvin. Three Generations: The Extreme Right in Finnish Politics. Bloomington, Indiana University Press, 1962.

SÉRANT, Paul. Les Dissidents de l'Action française. Paris, Copernic, 1978. [Études sur Georges Valois, Louis Dimier, Jacques Maritain, Georges Bernanos, Robert Brasillach, Thierry Maulnier, Claude Roy]

SHENFIELD, Stephen D. Russian Fascism. Traditions, Tendencies, Movements. Armonk et Londres, M. E. Sharpe, 2001.

SOUCY, Robert. Le fascisme français 1924-1933. Paris, Presses universitaires de France, 1989.

SOUCY, Robert. French Fascism: The Second Wave, $1933-$ 1939. New Haven, Yale University Press, 1995.

STERN, Fritz. The Politics of Cultural Despair: A Study in the Rise of the Germanic Ideology. Garden City, Doubleday, 1965. STERNHELL, Zeev. La droite révolutionnaire, 1885-1914: les origines françaises du fascisme. Paris, Gallimard, 1997.

STERNHELL, Zeev. Ni droite ni gauche. L'Idéologie fasciste en France. Bruxelles, Complexe, 1987.

SUGAR, Peter F., dir. Native Fascism in the Successor States, 1918-1945. Santa Barbara, ABC-Clio, 1971.

VENNER, Dominique. Histoire d'un fascisme allemand. Les corps-francs du Baltikum et la Révolution conservatrice, 19181933. Paris, Pygmalion, 1996.

WEBER, Eugen. Action française. Stanford, Stanford University Press, 1962. 
WEBER, Eugen. Varieties of Fascism. Doctrines of Revolution in the Twentieth Century. Princeton, Van Nostrand, 1964 (réimpression: Malabar, Robert E. Krieger Publishing Company, 1982).

WEBSTER, Richard A. The Cross and the Fasces. Christian Democracy and Fascism in Italy. Stanford, Stanford University Press, 1960.

WEISS, John. The Fascist Tradition. Radical Right-Wing Extremism in Modern Europe. New York, Harper \& Row, 1967. (Coll. "Major Traditions of World Civilization»).

WHITESIDE, Andrew G. Austrian National Socialism before 1918. La Haye, Martinus Nijhoff, 1962.

WINOCK, Michel, dir. Histoire de l'extrême droite en France. Paris, Seuil, 1993.

WOODS, Roger. The Conservative Revolution in the Weimar Republic. New York, St. Martin's Press, 1996.

WOOLF, Stuart J., dir. European Fascism. Londres, Weidenfeld \& Nicolson, 1968 